\title{
Fourier descriptors for shape: Effects of adaptation on discrimination thresholds
}

\author{
JOHN UHLARIK \\ Kansas State University, Manhattan, Kansas
}

\begin{abstract}
Fourier descriptors (FDs) have been used by investigators of computer pattern recognition to discriminate and/or identify planar shapes. These descriptors enable one to analyze and synthesize closed curves by obtaining Fourier coefficients of chain-encoded contours. Discrimination thresholds were obtained for FD stimuli for shape in a manner similar to contrast-sensitivity functions for grating stimuli. Adaptation to a specific FD harmonic frequency produced a selective decrease in sensitivity (i.e., increase in threshold) for the adapting frequency and for closely related, even harmonic frequencies. These results suggest that FDs are an important and promising tool for research on human perception of form.
\end{abstract}

The present research represents a preliminary attempt to determine the extent to which Fourier descriptors (FDs) for planar shape provide a relevant dimension for describing how the human visual system processes form. FDs represent transforms of boundary curvature that are popular in computer pattern recognition, because they are relatively easy to program and are backed by the welldeveloped Fourier mathematical theory (Pavlidis, 1980). These descriptors provide for both the analysis and the synthesis of closed curves by means of obtaining Fourier coefficients of chain-encoded contours. This approach permits a curve to be represented parametrically as a function of arc length, according to the accumulated change in the direction of the curve from its starting point. This function is expanded in a Fourier series, and the coefficients are arranged in the amplitude/phase angle form (cf. Kuhl \& Giardina, 1982; Zahn \& Roskies, 1972). Any two-dimensional shape can thus be fully described by its set of FDs; the low-frequency terms provide the global properties of the shape, and the addition of higherfrequency terms fills in more and more local detail.

Physiological evidence suggests that neurons in the striate cortex of infrahumans are selective for stimuli of specific boundary curvature (Schwartz, Desimone, Albright, \& Gross, 1983). If features such as FDs are involved in the coding of shape in the human visual system, they might exhibit selective adaptation too. The purpose in the present study was, first, to determine threshold functions for FD stimuli in a manner similar

This research was supported by the USAF-UES Summer Faculty Research Program, which was sponsored by the Air Force Systems Command and the Air Force Office of Scientific Research. Much of the impetus for the research came from George Geri and the resources provided by the Human Resources Laboratory at Williams Air Force Base in Arizona. The views of the author do not purport to reflect those of the United States Air Force. The author is extremely grateful to Chris Voltz for computer programming support. Correspondence may be addressed to John Uhlarik, Department of Psychology, Kansas State University, Manhattan, KS 66506. to how they are obtained for contrast sensitivity in the spatial-frequency domain (Davidson, 1968), and, second, to determine if these thresholds can be selectively elevated by means of the adaptation paradigm.

\section{METHOD}

\section{Stimuli and Design}

Stimulus patterns were generated with an algorithm developed by Kuhl and Giardina (1982). An arbitrarily chosen closed contour was used as a source (shown in Figure 1A), and this shape was chain encoded in order to obtain Fourier coefficients. Figure 1B shows the chain-code link labels that approximate the continuous contour in a sequence of piecewise linear fits (cf. Freeman, 1974). The Fourier series representation is appropriate for the chain code, because the code repeats on successive traversals of the contour, and because each term in the Fourier expansion is a descriptor associated with a particular frequency (defined in units of cycles/perimeter), amplitude (defined as the square root of the sum of the squared coefficients), and relative phase angle that can be manipulated orthogonally. The resulting FDs are invariant with

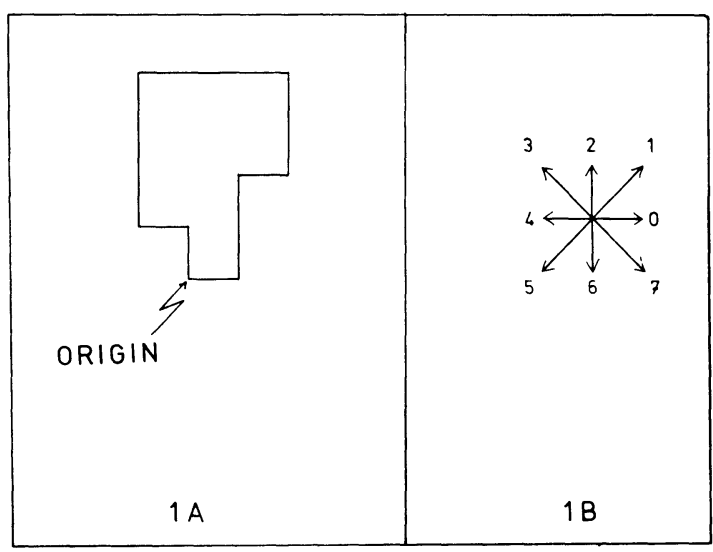

Figure 1. Figure 1A shows the source figure used to derive the Fourier-descriptor stimuli. Figure 1B shows the eight standardized line segments used to chain encode the source figure. Thus, from the point of origin, the chain code for this particular source figure would be: $2,2,2,2,2,4,4,4,4,4,2,2,2,2,2,2,2,2,2,2,0$, $0,0,0,0,0,0,0,0,0,6,6,6,6,6,6,6,6,6,6,4,4,4,4,4,6$, $6,6,6,6,6,6,6,6,6,4,4,4,4,4$. 
respect to rotation, dilation, and translation of the source contour, but they lose no information about the contour's shape (Kuhl \& Giardina, 1982).

By means of the psychophysical method of constant stimuli, observers were required to judge whether two FD shapes appeared to be the "same" or "different." Discrimination thresholds were obtained through determination of how much FD amplitude had to be associated with a shape of a given harmonic frequency for that frequency to be discriminated from the fundamental frequency $50 \%$ of the time. The fundamental was used as the standard and was presented on every trial. This firstorder harmonic was always associated with a fixed amplitude that determined its size. In the present case, the fundamental was an ellipse sharing only the most global shape and size of the source shape shown in Figure 1A. Comparison figures consisted of the sum (Fourier synthesis) of the fundamental and another higher order harmonic frequency, which could be varied in FD amplitude.

Ten different higher order, harmonic frequencies $(2,3,4,5,6,7$, $8,11,16,21)$ were used for the comparison figures, and each comparison figure could be associated with each of seven different harmonic amplitudes $(0.0,0.1,0.2,0.3,0.4,0.5,0.6)$. Figure 2 illustrates examples of the 10 harmonic frequencies used for comparison stimuli; this figure shows each comparison frequency associated with three different levels of amplitude. When the higher order frequency component of the comparison figure was associated with 0.0 amplitude, this frequency made no contribution to the comparison figure, and the comparison was identical to the standard in that both shapes consisted only of the fundamental harmonic frequency (i.e., an ellipse). However, when the higher order frequency was associated with a nonzero FD amplitude, it made a "real" contribution to the comparison figure, and the standard and comparison were in fact different. Thus, the discrimination threshold for each harmonic frequency was defined as the amplitude that caused the comparison figure to appear different from the standard $50 \%$ of the time.

A block of 120 trials consisted of 60 same and 60 different trials presented in random order. Within each block, the higher order harmonic component of the comparison figure was always the same frequency but was varied in amplitude. For the 60 same trials, both figures consisted of the fundamental harmonic frequency in that the higher order component of the comparison was associated with zero amplitude. For the 60 different trials, the comparison figures consisted of 10 presentations each of the six nonzero amplitudes (i.e., $0.1,0.2,0.3,0.4,0.5$, 0.6). A complete replication consisted of 10 blocks ( 1 for each higher order harmonic frequency) for each observer.

The stimuli were generated and displayed on an IBM AT computer with an IBM 5175 Professional Graphics System. The horizontal resolution was 640 pixels; vertical resolution was 480 pixels. A small fixation cross was located at the center of the display. The standard and comparison figures were filled closed contours that were centered at points $.75^{\circ}$ to the left and right of fixation; each figure subtended $.75^{\circ}$ visual angle. On half of the trials, the standard figure was on the left side of each pair, and on the other half it was on the right.

\section{Procedure}

The observers viewed the display binocularly from a distance of $1.45 \mathrm{~m}$. The observers were instructed to fixate a small cross at the center

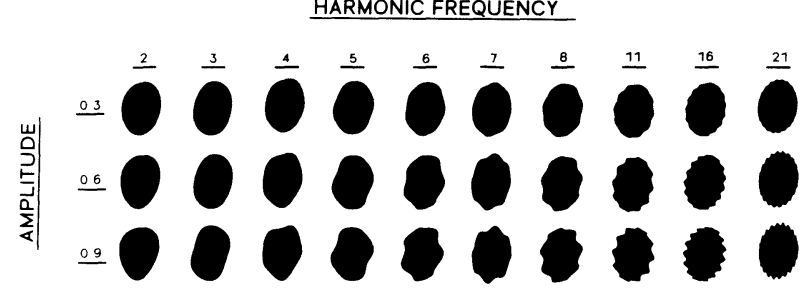

Figure 2. Examples of the comparison figures used as the independent variables for harmonic frequency and amplitude. Individual figures consisted of the fundamental harmonic frequency summed with a higher order harmonic frequency. Three levels of Fourierdescriptor amplitude are illustrated for each frequency. of the screen and not to make gross eye movements on each trial until the trial was completed. A noise screen consisting of a random-dot pattern (50\% of the pixels illuminated) was always present on the display, except during presentation of the target and adapting stimuli. The purpose of the noise screen was to eliminate persistence of the target and adapting stimuli and to reduce contour masking. The space-average luminance of the noise screen was $0.2 \mathrm{fL}$; the luminance of the adapting and target figures was $0.62 \mathrm{fL}$.

Initially, basic threshold data were obtained for all observers. A threshold trial consisted of simultaneous presentation for $150 \mathrm{msec}$ of a pair of target stimuli (i.e., the standard and the comparison). The noise screen preceded and followed the presentation of the pair of target stimuli. If the two target stimuli appeared to be identical in shape, the observer pressed a switch indicating "same"; if the pair did not appear to be identical, the observer pressed a second switch, indicating "different." Following each trial, the observers received auditory feedback regarding the correctness of their choices. A 0.5 -sec warning tone preceded each trial by $0.5 \mathrm{sec}$. The trials were initiated by the observers. For the initial threshold data, there were two complete replications of all 10 blocks (harmonic frequencies) for each observer. Different random orders of blocks were used for each subject. Typically, an experimental session consisted of 2 or 3 blocks, and two or three sessions were run daily. All observers were given 3 practice blocks prior to the start of the actual experiment.

Subsequent to determining basic thresholds, adaptation data were collected. The sequence for an adaptation trial was similar to that for a threshold trial, but with the addition of preexposure, for $2 \mathrm{sec}$, to a pair of high-amplitude adapting stimuli. After the 2-sec exposure to the adapting stimuli, the noise screen came on for an interval of $1 \mathrm{sec}$, which was in turn followed by the $150-\mathrm{msec}$ presentation of the target stimuli. The adapting stimuli were always an identical pair of shapes consisting of the fundamental frequency summed with the sixth harmonic. The amplitude of the sixth harmonic used for adaptation was 1.5 , which was approximately six times the amplitudes associated with the $50 \%$ amplitude-discrimination thresholds. Adaptation blocks for Harmonic Frequencies 4, 5, 6, 7, and 8 were replicated twice for each observer; adaptation blocks for Frequencies 2, 3, 11, 16, and 21 were replicated once for each observer.

\section{Subjects}

Three male observers participated in the experiment. Two observers had emtropic vision, and the other (T.H.) had vision corrected to 20/20 for the viewing conditions involved in the experiment. Two of the observers (T.H. and J.N.) were aware of the general purpose and rationale of the experiment, but they were naive regarding specifics of the design such as the order of the blocks. J.U. (the author) was aware of such details while serving as a subject.

\section{RESULTS}

When the higher order component of the comparison figure had an amplitude of $0.1,0.2,0.3,0.4,0.5$, or 0.6 , the comparison and standard figures were in fact different. In any given block of 120 trials, each of the nonzero amplitudes was presented 10 times, thus constituting 60 different trials. The amplitude-discrimination threshold for each block was defined as the FD amplitude that the observer reported as different $50 \%$ of the time. In Figure 3, this discrimination amplitude is plotted as a function of harmonic frequency for each of the 3 observers. Note, however, that in Figure 3 the vertical axis is reversed so that it displays the curves in terms of relative sensitivity, and hence they are analogous to contrast-sensitivity functions in the spatial-frequency domain (cf. Cornsweet, 1970). Figure 3 shows that these functions appear to have the same overall shape for the 3 observers; generally, sensitivity is low (i.e., amplitude-discrimination thresholds 
are high) at the low harmonic frequencies, it is maximal for Frequencies 4 through 7, and it declines somewhat at higher harmonic frequencies. The effects of adaptation for each observer are shown in Figure 4. This figure shows that for each observer, there was an elevation of threshold for the sixth harmonic frequency, which of

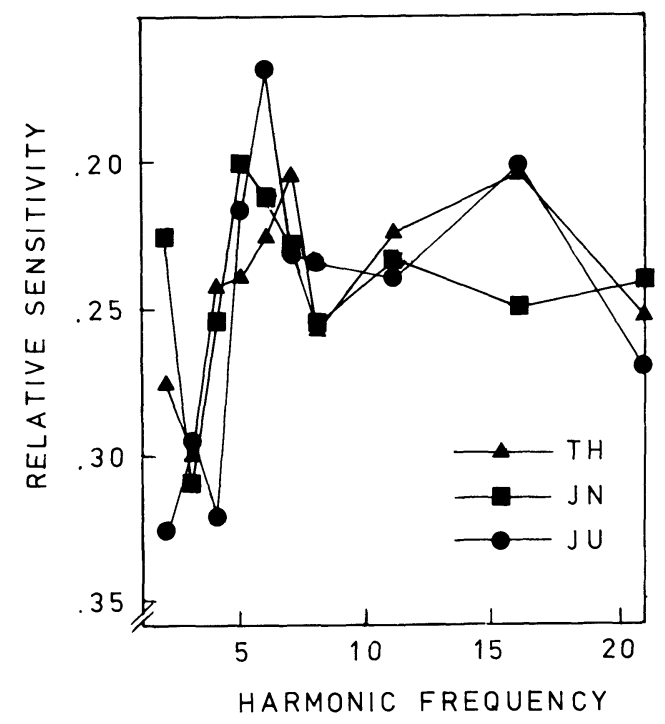

Figure 3. Fourier amplitude-discrimination sensitivity (no adaptation) as a function of harmonic frequency, for the three individual observers. (Note that the vertical axis is reversed so that these functions represent sensitivity rather than thresholds per se, and are analogous to contrast-sensitivity functions in the spatial-frequency domain.)

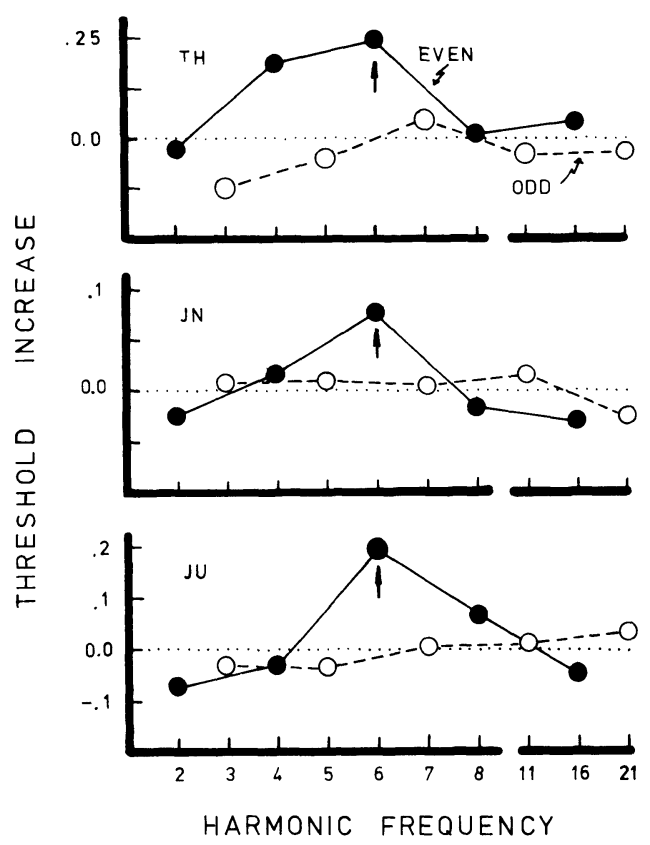

Figure 4. Increase in amplitude-discrimination thresholds, as a function of harmonic frequency subsequent to adaptation to the sixth harmonic frequency.

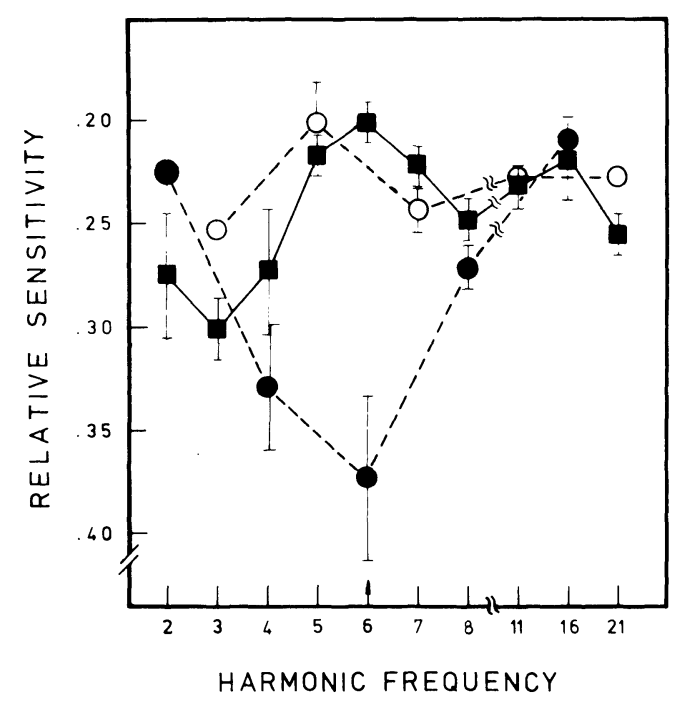

Figure 5. Basic Fourier amplitude-threshold sensitivity, and the effects of adaptation, averaged over the three observers. The filled squares connected with solid lines represent sensitivity functions obtained with no adaptation. The circles represent adaptation conditions involving preexposure to the sixth harmonic. The filled circles are for even, the open circles for odd harmonic frequencies. (Again the vertical axis is reversed, so that the greater the sensitivity, or the lower the threshold, the higher the position on the vertical axis.)

course corresponded to the harmonic frequency of the adapting stimuli. This figure also suggests that the effects of adaptation to the sixth harmonic spread to the adjacent even harmonics but had no systematic influence on the odd harmonic frequencies.

Figure 5 summarizes these same effects averaged over the 3 observers. The basic threshold data involving no adaptation are represented by filled squares connected with solid lines. Again the vertical axis is reversed, so that the effects are displayed in terms of relative sensitivity rather than thresholds per se. As was the case in Figure 3, this curve indicates that sensitivity is relatively low for the low harmonic frequencies, that it is greatest in the midrange around the fifth, sixth, and seventh harmonics, and that it displays a small decline at the higher harmonics. Figure 5 also shows the effects of adaptation to the highamplitude sixth harmonic frequency, averaged over the 3 observers. These adaptation effects are plotted separately for the even and odd harmonic frequencies; in both cases, these curves are represented by circles connected with dashed lines (the filled circles represent the even, and the open circles represent the odd harmonic frequencies). Figure 5 shows that adaptation to the sixth harmonic produced a pronounced decrease in relative sensitivity (i.e., increase in threshold) for the sixth harmonic frequency; adjacent even harmonics (i.e., the fourth and the eighth) also led to some impairment in relative sensitivity. However, the dashed curve connecting the open circles in Figure 5 indicates that the odd harmonics were unaffected by adaptation to the sixth harmonic frequency. 


\section{DISCUSSION}

The results of the present research suggest that FDs are an importan tool for research on human perception of form. A persistent problem in the study of form perception and pattern recognition has been the lack of a complete and reliable measure of the physical stimulus. Voluminous research has addressed this fundamental issue, but reviews indicate that it has never been resolved (e.g., Brown \& Owen, 1967; Michels \& Zusne, 1965; Treisman, 1986). Fourier descriptors have the potential to provide such a measure, because they are a powerful and efficient algorithm for representing and classifying shapes.

To the extent that the adaptation paradigm provides an indication of neural coding, the finding that prolonged exposure to a specific harmonic frequency elevates threshold in the region of that frequency suggests that the processing of form in the human visual system may involve the decomposition of shape into FDs related to boundary curvature. Further research is needed to determine if these adaptation effects occur for other adapting frequencies, how much they generalize when other source figures are used to generate the FD stimuli, and whether there are systematic effects of adaptation on the odd as opposed to even harmonic frequencies.

\section{REFERENCES}

BRown, D. R., \& OWEN, D. H. (1967). The metrics of visual form: Methodological dyspepsia. Psychological Bulletin, 68, 243-259.
Cornsweet, T. N. (1970). Visual perception. New York: Academic Press.

Davidson, M. L. (1968). Perturbation approach to spatial brightness interaction in human vision. Journal of the Optical Society of America, 58, 1300-1309.

FreEMAN, H. (1974). Computer processing of line drawing images. Computing Survey, 6, 57-97.

KuHL, F. P., \& Giardina, C. R. (1982). Elliptic Fourier features of a closed contour. Computer Graphics \& Image Processing, 18, 230-258.

Michels, K. M., \& Zusne, L. (1965). Metrics of visual form. Psychological Bulletin, 63, 74-86.

PAvilidis, T. (1980). Algorithms for shape analysis of contours and waveforms. Transactions on Pattern Analysis \& Machine Intelligence, 2, 301-312.

Schwartz, E. L., Desimone, R., Albright, T. D., \& Gross, C. G. (1983). Shape recognition and inferior temporal neurons. Proceedings of the National Academy of Sciences, 80, 5776-5778.

Treisman, A. (1986). Properties, parts and objects. In K. R. Boff, L. Kaufman, \& J. P. Thomas (Eds.), Handbook of visual perception (Vol. II, pp. 35.1-35.70). New York: Wiley.

ZAHN, C. T., \& Roskies, R. Z. (1972). Fourier descriptors for plane closed curves. IEEE Transactions on Computers, C-21, 296-281.

(Manuscript received December 22, 1988.) 